\title{
2-D DOA Estimation of LFM Signals based on Sparse Representation in Fractional Fourier Domain
}

\author{
Zhibo Shen, Songyang Liu, Yangyang Dong, Guoqing Zhao, Chunxi Dong \\ Key Laboratory of Electronic Information Countermeasure and Simulation Technology, Ministry of Education, Xidian University, \\ Xi'an 710071, Shaanxi, China \\ E-mail: youfenglaiyi1026@163.com
}

\begin{abstract}
For the problem of two dimensional direction of arrival (2-D DOA) estimation of Liner Frequency Modulated (LFM) signals, a novel 2-D DOA estimation method is proposed based on sparse representation in fractional Fourier domain. First, utilizing the energy-concentrated characteristic of fractional Fourier transform for LFM signals, the redundant dictionaries based on the space angles are established in fractional Fourier domain. Then the space angles can be estimated by sparse recovery algorithm respectively. Finally, the azimuth and elevation angles are calculated through the estimated space angles. The proposed method has a better estimation precision, high-resolution performance and lower SNR threshold without multidimensional search process. The validity of the proposed method is verified by theoretic analysis and simulation results.
\end{abstract}

Keywords-LFM signal; DOA estimation; fractional Fourier transform; sparse recovery

\section{INTRODUCTION}

Linear frequency modulation (LFM) signal is widely used in a number of fields including radar, sonar and communications [1]. The direction of arrival (DOA) of LFM signal, which is a crucial parameter for sorting and recognition of sources, directing jamming and passive location, has attracted tremendous interest in electronic reconnaissance. The conventional subspace-type DOA estimation methods based on the wide-sense stationary signal model dominate in the literature due to their high-resolution performance [2-3]. However, these high-resolution methods cannot be applied to the LFM signal directly, which is a typical non-stationary signal. Therefore, how to estimate the two dimension (2-D) DOA of LFM signals is an important problem. In [4], Gershman utilizes interpolation in spatial time-frequency distribution matrices to realize DOA estimation for LFM signals. This approach suffers from the disturbance of crossterms and exist the model biases and time consuming. The fractional Fourier transform (FRFT), which is a generalization of the classical Fourier transform, is proposed in [5]. Almeida proves the FRFT's relationships with time-frequency representations [6], which made FRFT especially suitable for the energy-concentration of LFM signals. Tao attempted to change the received data of sensor array into fractional Fourier domain, and then estimated the 2-D DOAs by subspace algorithms (like MUSIC or ESPRIT) [7]. However, this method needs two-dimensional search process in angle space, which leads to large amount of computation. In recent years, the emerging field of sparse representation has given renewed interest to the problem of DOA estimation [8-9]. These methods try to find a sparse spatial spectrum from the dictionary by solving a sparse recovery problem. For 2-D DOA estimation, the dictionary should be capable of containing all the possible azimuth and elevation angles. To improve the spatial intercept probability, the reconnaissance receiver tends to cover the whole spatial domain, which results in a large dictionary. The sparse recovery will becomes more difficult and complicated with the growth of dictionary length.

In this paper, a novel 2-D DOA estimation method of LFM signals based on sparse representation in fractional Fourier domain is proposed. Utilizing the excellent energyconcentrated performance of LFM signal in the fractional Fourier domain [10-11], the received signals of sensor array are transformed into fractional Fourier domain, which changes the time-variant manifold matrices of LFM signals into timeinvariant one. Then, two 1-D dictionaries are established respectively based on the defined space angle. The problem of 2-D DOAs estimation is converted into the problem of two 1-D DOAs estimation, which reduces the length of dictionary. In this case, a considerable amount of computations can be saved while estimating the space angles by sparse recovery algorithm. Once the space angles are known, azimuth and elevation angles can be achieved by simple calculations. Theoretical analyses and simulations show that the proposed method has better estimation precision and high-resolution performance without multidimensional search process in spatial domain.

\section{THE ARRAY MODEL}

An L-shape array configuration in the $x$-y plane is composed of two orthogonal uniform linear arrays with interelement spacing $d$ as shown in Fig.1. The element placed at origin is common for referencing purpose. Let $X$ be the subarray of the linear array in the $x$ axis, and let $Y$ be the sub-array of the linear array in the $y$ axis, where each linear array consists of $N$ - 1 elements. 


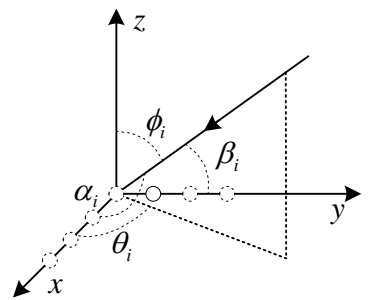

Fig. 1. L-shape uniform linear array with $N-1$ sensors in the sub-array $X$ and $Y$ respectively.

Assume that there are $p$ far-field LFM signals impinging on the array from directions $\left(\theta_{1}, \phi_{1}\right),\left(\theta_{2}, \phi_{2}\right), \ldots,\left(\theta_{p}, \phi_{p}\right)$, where the $i$ th source has an azimuth $\theta_{i}$ and an elevation $\phi_{i}$, $i=1,2, \ldots, p$. The received data at the $n$th sensor of sub-array $X$ and $Y$ is expressed as

$$
\left\{\begin{array}{l}
x_{n}(t)=\sum_{i=1}^{p} s_{i}\left(t-\tau_{x_{i, n}}\right)+n_{x_{n}}(t) \\
y_{n}(t)=\sum_{i=1}^{p} s_{i}\left(t-\tau_{y_{i, n}}\right)+n_{y_{n}}(t)
\end{array}, n=0,1, \ldots N-1\right.
$$

where

$$
\begin{aligned}
\tau_{x_{i, n}} & =\frac{(i-1)}{c} d \cos \theta_{i} \sin \phi_{i} \\
\tau_{y_{i, n}} & =\frac{(i-1)}{c} d \sin \theta_{i} \sin \phi_{i} \\
s_{i}(t) & =\exp \left[\mathrm{j} 2 \pi\left(f_{i} t+\mu_{i} t^{2} / 2\right)\right]
\end{aligned}
$$

where $\tau_{x_{i, n}}$ and $\tau_{y_{i, n}}$ are the propagation delays for the $i$ th source between the reference sensor and the $n$th sensor in sub-array $X$ and $Y . f_{i}$ and $\mu_{i}$ denote respectively the initial frequency and chirp-rate of $i$ th LFM signal. $n_{x_{n}}(t)$ and $n_{y_{n}}(t)$ are the white Gaussian noise at the $n$th sensor in sub-array $X$ and $Y$ respectively, which is assumed to be statistically independent with signals. Let $\alpha_{i}$ be the angle between the directions of $i$ th source and $x$ axis, and let $\beta_{i}$ be the angle between the directions of $i$ th source and $y$ axis. By the spatial geometric position relation, we can obtain

$$
\left\{\begin{array}{l}
\cos \alpha_{i}=\cos \theta_{i} \sin \varphi_{i} \\
\cos \beta_{i}=\sin \theta_{i} \sin \varphi_{i}
\end{array}\right.
$$

Combining (4) and (2), we have that

$$
\left\{\begin{array}{l}
\tau_{x_{i, n}}=\frac{(n-1)}{c} d \cos \alpha_{i} \\
\tau_{y_{i, n}}=\frac{(n-1)}{c} d \cos \beta_{i}
\end{array}\right.
$$

From (4)-(5), it is obvious that azimuth $\theta_{k}$ and elevation $\phi_{k}$ can be represented by space angle $\alpha_{i}$ and $\beta_{i}$. In this case, the azimuth and elevation estimation is converted into the space angle estimation, which is the problem of two 1-D DOAs estimation.

\section{THE PROPOSED METHOD}

Through the definition of space angle, the problem of 2-D DOAs estimation is converted into the problem of two 1-D DOAs estimation. However, due to the wideband and nonstationary properties of LFM signals, the existing methods based on narrowband model cannot be applied directly. Considering the FRFT's energy-concentrated performance for LFM signal, we can deal with it in fractional Fourier domain.

\section{A. Fractional Fourier transform(FRFT)}

According to the definition of FRFT, the FRFT of the $i$ th source at the reference sensor is

$$
\begin{aligned}
& S_{i, 0}(\eta, u)=\sqrt{\frac{1-\mathrm{j} \cot \eta}{2 \pi}} \exp \left(\mathrm{j} \frac{u^{2}}{2} \cot \eta\right) \\
& =\int_{-T / 2}^{T / 2} \exp \left[\mathrm{j} \frac{t^{2}}{2}\left(\cot \eta+\mu_{i}\right)-\mathrm{j} t\left(u \csc \eta-f_{i}\right)\right] d t
\end{aligned}
$$

where $\mathrm{q}$ is the transform order of the FRFT, $\eta=q \pi / 2$ and $\mathrm{T}$ is the observation time. From (6), it can be seen that, if $\eta_{i 0}=-\cot \mu_{i}$, the equation (6) can be written as

$$
\begin{aligned}
& S_{i, 0}(\eta, u)=\sqrt{1-\mathrm{j} \cot \eta_{i 0}} \exp \left(\mathrm{j} \pi u^{2} \cot \eta_{i 0}\right) \\
& T \frac{\sin \left(\pi\left(u \csc \eta_{i 0}-f_{i 0}\right) T\right)}{\pi\left(u \csc \eta_{i 0}-f_{i 0}\right) T}
\end{aligned}
$$

we can prove that, $S_{i, 0}(\eta, u)$ has the best energy-concentrated performance when $\eta_{i, 0}=-\cot \mu_{i}$ [12]. There will be a spectral peak on the $(\eta, u)$ plane with the position and value given by

$$
\begin{gathered}
u_{i 0}=f_{i 0} / \csc \eta_{i 0} \\
S_{i, 0}\left(\eta_{i 0}, u_{i 0}\right)=\sqrt{1-\mathrm{j} \cot \eta_{i 0}} \exp \left(\mathrm{j} \pi u_{i 0}^{2} \cot \eta_{i 0}\right) T
\end{gathered}
$$

Then, the initial frequency $f_{i}$ and chirp-rate $\mu_{i}$ of ith LFM signal is estimated by

$$
\left\{\begin{array}{l}
\hat{f}_{i}=u_{i 0} \csc \eta_{i 0} \\
\hat{\mu}_{i}=-\cot \eta_{i 0}
\end{array}\right.
$$

For LFM signals, the propagation delay has no influence on the transform order of the FRFT. The $i$ th LFM signal at the reference sensor and its time delay version at the nth sensor of sub-array $X$ or $Y$ achieves energy concentration at the same order. Therefore, we have that

$$
\left\{\begin{array}{l}
S_{x_{i, n}}\left(\eta_{i 0}, u_{x_{i, n}}\right)=B_{i}\left(\tau_{x_{i, n}}\right) \sqrt{1-\mathrm{j} \cot \eta_{i 0}} \exp \left(\mathrm{j} \pi u_{x_{i, n}}^{2} \cot \eta_{i 0}\right) T \\
S_{y_{i, n}}\left(\eta_{i 0}, u_{y_{i, n}}\right)=B_{i}\left(\tau_{y_{i, n}}\right) \sqrt{1-\mathrm{j} \cot \eta_{i 0}} \exp \left(\mathrm{j} \pi u_{y_{i, n}}^{2} \cot \eta_{i 0}\right) T
\end{array}\right.
$$

where

$$
\begin{gathered}
B_{i}\left(\tau_{x(y)_{i, n}}\right)=\exp \left[\mathrm{j} \pi\left(-2 f_{i} \tau_{x(y)_{i, n}}+\mu_{i} \tau_{x(y)_{i, n}}^{2}\right)\right] \\
u_{x(y)_{i, n}}=u_{i 0}+\tau_{x(y)_{i, n}} \cos \eta_{i 0}
\end{gathered}
$$

where $u_{x_{i, n}}$ and $u_{y_{i, n}}$ denote the position of spectral peak in fractional Fourier domain for the ith LFM signal at the nth sensor of sub-array $X$ and $Y$ respectively.

From the discussion above, we conclude that the same LFM signal received by different sensors from different sub- 
arrays $(X$ or $Y$ ) will achieve the best energy-concentrated performance at the same transform order of the FRFT and the position of spectral peak is due to the propagation delay $\tau_{x_{i, n}}, \tau_{y_{i, n}}$ at different sensors.

\section{B. Sparse representation based on fractional Fourier domain}

For different LFM signals, the peaks of them in fractional Fourier domain appear in different transform orders and positions. As these peak values dominate most of the energy, they are selected as the array received data. Substituting (14) into (11), it can be rewritten as

$$
\left.\begin{array}{l}
S_{x_{i, n}}\left(\eta_{i 0}, u_{x_{i, n}}\right)=\sqrt{1-\mathrm{j} \cot \eta_{i 0}} \exp \left[-\mathrm{j} \pi\left(\tau_{x_{i, n}}^{2} \sin \eta_{i 0} \cos \eta_{i 0}\right.\right. \\
\left.\left.+2 u_{i 0} \tau_{x_{i, n}} \sin \eta_{i 0}\right)\right] \exp \left(\mathrm{j} \pi u_{i 0}^{2} \cot \eta_{i 0}\right) T \\
S_{y_{i, n}}\left(\eta_{i 0}, u_{y_{i, n}}\right)=\sqrt{1-\mathrm{j} \cot \eta_{i 0}} \exp \left[-\mathrm{j} \pi\left(\tau_{y_{i, n}}^{2} \sin \eta_{i 0} \cos \eta_{i 0}\right.\right. \\
\left.\left.+2 u_{i 0} \tau_{y_{i, n}} \sin \eta_{i 0}\right)\right] \exp \left(\mathrm{j} \pi u_{i 0}^{2} \cot \eta_{i 0}\right) T
\end{array}\right\}
$$

where $\tau_{x_{i, n}}^{2}$ and $\tau_{y_{i, n}}^{2}$ are small, and can be ignored. Combining (5) and (9), equation (14) can be rewritten as

$$
\begin{aligned}
& S_{x_{i, n}}\left(\eta_{i 0}, u_{x_{i, n}}\right)=\exp \left[-\mathrm{j} 2 \pi u_{i 0}(n-1) d\right. \\
& \left.\cos \alpha_{i} \sin \eta_{i 0} / c\right] S_{i, 0}\left(\eta_{i 0}, u_{i 0}\right)=a_{x_{i, n}} S_{i, 0}\left(\eta_{i 0}, u_{i 0}\right) \\
& S_{y_{i, n}}\left(\eta_{i 0}, u_{y_{i, n}}\right)=\exp \left[-\mathrm{j} 2 \pi u_{i 0}(n-1) d\right. \\
& \left.\cos \beta_{i} \sin \eta_{i 0} / c\right] S_{i, 0}\left(\eta_{i 0}, u_{i 0}\right)=a_{y_{i, n}} S_{i, 0}\left(\eta_{i 0}, u_{i 0}\right)
\end{aligned}
$$

where

$$
\left.\begin{array}{l}
a_{x_{i, n}}=\exp \left[-\mathrm{j} 2 \pi u_{i 0}(n-1) d \cos \alpha_{i} \sin \eta_{i 0} / c\right] \\
a_{y_{i, n}}=\exp \left[-\mathrm{j} 2 \pi u_{i 0}(n-1) d \cos \beta_{i} \sin \eta_{i 0} / c\right]
\end{array}\right\}
$$

From (15), we can see that the propagation delays at sensors of sub-array $X$ and $Y$ are only related to space angle $\alpha_{i}$ and $\beta_{i}$ after the LFM signal is transformed into fractional Fourier domain. As sub-array $X$ and $Y$ are mutually independent, the steering vector of $i$ th source in fractional Fourier domain can be respectively defined as

$$
\left.\begin{array}{l}
a_{x_{i, n}}=\exp \left[-\mathrm{j} 2 \pi u_{i 0}(n-1) d \cos \alpha_{i} \sin \eta_{i 0} / c\right] \\
a_{y_{i, n}}=\exp \left[-\mathrm{j} 2 \pi u_{i 0}(n-1) d \cos \beta_{i} \sin \eta_{i 0} / c\right]
\end{array}\right\}
$$

Thus, we can establish the redundant dictionaries respectively in fractional Fourier domain, utilizing the steering vectors with uniform discrete cosines of space angle $\alpha_{i}$ and $\beta_{i}$. The cosines of all potential space angles are divided into discrete sets. Let the set $(\cos \alpha(\beta))_{m}=-1+2 m / N_{s}$, where $N_{s}$ being the number of scanning cosine. Combining the steering vector given by (17), the dictionaries of size $N \times N_{s}$ in a certain transform order of FRFT is expressed as

$$
\left\{\begin{array}{l}
\boldsymbol{D}_{\eta_{i 0}, u_{i 0}}^{(X)}(\alpha)=\left[\boldsymbol{a}_{X}\left(\alpha_{1}\right), \boldsymbol{a}_{X}\left(\alpha_{2}\right), \ldots, \boldsymbol{a}_{X}\left(\alpha_{N_{s}}\right)\right] \\
\boldsymbol{D}_{\eta_{i 0}, u_{i 0}}^{(Y)}(\beta)=\left[\boldsymbol{a}_{Y}\left(\beta_{1}\right), \boldsymbol{a}_{Y}\left(\beta_{2}\right), \ldots, \boldsymbol{a}_{Y}\left(\beta_{N_{s}}\right)\right]
\end{array}\right.
$$

where $\eta_{i 0}$ denotes the transform order. Utilizing the dictionaries from (17), the data vector received at sub-array $X$ and $Y$ can be sparse representation, whose sparse representation in fractional Fourier domain is shown as (19)

$$
\left\{\begin{array}{l}
\boldsymbol{X}_{\eta_{i 0}}=\boldsymbol{D}_{\eta_{i 0}, u_{i 0}}^{(X)}(\alpha) z_{X}+\boldsymbol{N}_{X} \\
\boldsymbol{Y}_{\eta_{i 0}}=\boldsymbol{D}_{\eta_{i 0}, u_{i 0}}^{(Y)}(\beta) z_{Y}+\boldsymbol{N}_{Y}
\end{array}\right.
$$

where $\boldsymbol{X}_{\eta_{i 0}}=\left[x_{\eta_{i 0}, u_{i 0}}, \ldots, x_{\eta_{i 0}, u_{i, N-1}}\right]^{\mathrm{T}}, \quad \boldsymbol{Y}_{\eta_{i 0}}=\left[y_{\eta_{i 0}, u_{i 0}}, \ldots, y_{\eta_{i 0}, u_{i, N-1}}\right]^{\mathrm{T}}$ respectively denote the $N \times 1$ vector composed of the array received data under $\eta_{i 0}$ order of FRFT, which the peak values are selected as the observed data for each sensor in sub-array $X$ and $Y . z_{X}$ and $z_{Y}$ denote the $N_{s} \times 1$ sparse vector with non-zero elements at positions corresponding to the space angles and zero elements at the remaining positions. $\boldsymbol{N}_{X}$ and $\boldsymbol{N}_{Y}$ denote the $N \times 1$ vector representing the noise at sub-array $X$ and $Y$.

Estimating the space angles process is targeted at finding the positions of non-zero elements. We can treat this problem as a sparse recovery problem [13-14], i.e.,

$$
\min _{z}\|z\|_{1} \text { s.t. }\|\boldsymbol{D} \boldsymbol{z}-\boldsymbol{x}\|_{2}<\varepsilon
$$

where $z$ is the sparse vector, $\boldsymbol{D}$ is the dictionary and $\boldsymbol{x}$ is the received data vector. $\varepsilon$ denotes the reconstruction error. The support of $z$ can be obtained by conventional sparse recovery algorithms, such as Basis Pursuit (BP) [15], Orthogonal Matching Pursuit (OMP) [16], etc. Then, the space angles can be achieved through the positions of non-zero elements in $z_{X}$ and $z_{Y}$.

\section{Pair matching of azimuth and elevation angles}

As defining the space angle, the 2-D DOAs estimation problem is changed into two 1-D DOAs estimation problem. For calculating the azimuth $\theta_{i}$ and elevation $\phi_{i}$, it is necessary to determine the corresponding relation between space angles $\alpha_{i}$ and $\beta_{i}$. From the analysis above, the same LFM signal received by sub-array $\mathrm{X}$ and $\mathrm{Y}$ will achieve the best energy concentration at the same order and the estimated value of initial frequency $\hat{f}_{i}$ and chirp rate $\hat{\mu}_{i}$ calculated by (10) are also the same. Therefore, the pair matching problem of space angles can be effectively solved by $\hat{f}_{i}$ and $\hat{\mu}_{i}$, which is respectively estimated by the received data from sub-array $\mathrm{X}$ and Y. While the corresponding relation between space angles $\alpha_{i}$ and $\beta_{i}$ is determined, the azimuth $\theta_{i}$ and elevation $\phi_{i}$ can be calculated simply as follows

$$
\left\{\begin{array}{c}
\theta_{i}=\arctan \left(\frac{\cos \beta_{i}}{\cos \alpha_{i}}\right) \\
\varphi_{i}=\arcsin \sqrt{\cos ^{2} \alpha_{i}+\cos ^{2} \beta_{i}}
\end{array}, i=1,2, . . p\right.
$$

\section{Performance analysis}

The proposed method converts the 2-D DOA estimation problem into sparse recovery problem in fractional Fourier domain. By defining the space angle, this method set up 1-D dictionaries for sub-array $\mathrm{X}$ and $\mathrm{Y}$ respectively instead of the azimuth and elevation joint dictionary, which can reduce the length of dictionary effectively. For example, the length of 
azimuth and elevation joint dictionary is $N_{1} \times N_{2}$ while the length of space angle dictionary is only $N_{s}$. Assume that we utilize the OMP sparse recovery algorithm, whose computation is $O\left(k N_{L}\right)$, where $k$ denotes the sparsity ratio and $N_{L}$ denotes the length of dictionary. If we set $N_{1}=N_{2}=N_{s}=1000$, under the same sparsity ratio $\mathrm{k}$, the computation of sparse recovery is $O\left(k \cdot 10^{6}\right)$ with the azimuth and elevation joint dictionary but $O\left(k \cdot 10^{3}\right)$ with the space angle dictionary. Therefore, utilizing the 1-D space angle dictionaries will effectively decrease the computation of sparse recovery. In addition, the proposed method avoid the calculation of covariance matrix and eigenvalue decomposition process compared with the conventional subspace algorithms and has no use to conduct multidimensional search process in 2-D angle space.

\section{Simulation RESUlTS}

In this section, we provide several sets of simulation results to illustrate the performance of the proposed method for 2-D DOA estimation. In all the simulation examples below, we suppose that $\theta \in\left(-90^{\circ}, 90^{\circ}\right), \phi \in\left(0^{\circ}, 90^{\circ}\right)$ for all the sources. The length of redundant dictionary is set to $N_{s}=1000$. Since the number of peak values is known in a certain transform order, it can be treated as the sparse recovery problem with known sparsity ratio. The OMP sparse recovery algorithm is selected here.

\section{A. Simulation and settings}

Example 1: This simulation example considers the case where three uncorrelated LFM signals $(p=3)$ impinge on the Lshape array with initial frequencies $6 \mathrm{MHz}, 10 \mathrm{MHz}, 20 \mathrm{MHz}$ and chirp rate $1 \mathrm{MHz} / \mu \mathrm{s}, 1.2 \mathrm{MHz} / \mu \mathrm{s},-0.5 \mathrm{MHz} / \mu \mathrm{s}$. The total number of sensors is 15 , which each sub-array has 7 sensors. The number of snapshots $T$ and SNR are set to 512 and $15 \mathrm{~dB}$, respectively. $\left(-15^{\circ}, 25^{\circ}\right),\left(30^{\circ}, 5^{\circ}\right)$ and $\left(60^{\circ}, 15^{\circ}\right)$ are the true DOAs. Fig. 2 shows the space angle estimated results by sparse recovery. Fig. 3 shows the pair matching results of azimuth and elevation angles.

Example 2: In this simulation example, we consider the resolution performance of space angle. Choose two LFM signals, whose space angles are adjacent. The initial frequency are $10 \mathrm{MHz}, 8 \mathrm{MHz}$ and the chirp rate are $0.8 \mathrm{MHz} / \mu \mathrm{s}$, $1.1 \mathrm{MHz} / \mu \mathrm{s}$. Taking the sub-array $X$ as an example, space angles $\alpha$ are set to $30^{\circ}$ and $31^{\circ}$. The other simulation conditions are similar to those of Example 1. Fig. 4 shows the estimated results of MUSIC algorithm and proposed method.

Example 3: In this simulation example, we consider the influence of angle interval on RMSE of angle. Taking the subarray $X$ as an example, the number of sensors in sub-array $X$ is set to 4 and 8 respectively. Choose two LFM signals, whose initial frequency and chirp rate are the same as Example 1. Space angle of signal 1 is fixed at $\alpha_{1}=30^{\circ}$ and space angle of signal $2, \alpha_{2}$ varies from $31^{\circ}$ to $45^{\circ}$ with interval $1^{\circ}$. The other simulation conditions are similar to those of Example 1. Fig. 5 shows the RMSE of angles as a function of angle interval with different number of sensors in sub-array $X$, averaged over 1000 Monte Carlo trials.

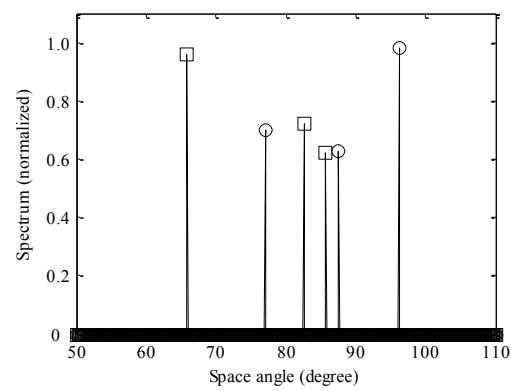

Fig. 2. Estimated results of space angles

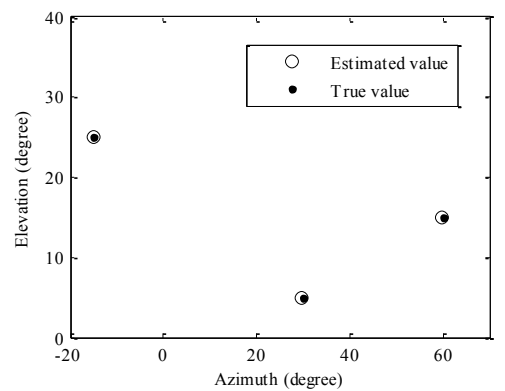

Fig. 3. Estimated results of 2-D DOAs

Example 4: In this simulation example, we consider the 2D DOA estimation performance with respect to SNR. The simulation conditions are similar to those of Example 1 except the SNR. The SNR varies from $-10 \mathrm{~dB}$ to $30 \mathrm{~dB}$ with interval $1 \mathrm{~dB}$. Every fixed frequency conducts 1000 Monte Carlo trials. The RMSE of angle as a function of SNR is shown in Fig. 6.

\section{B. Discussion and analysis}

From Fig. 2, we can see that the space angles can be estimated effectively by OMP sparse recovery algorithm. Fig. 3 gives the azimuth and elevation estimated results according to the estimated value of matched space angles, which proves that azimuth and elevation can be pair matched correctly by the initial frequencies and chirp rates of different signals.

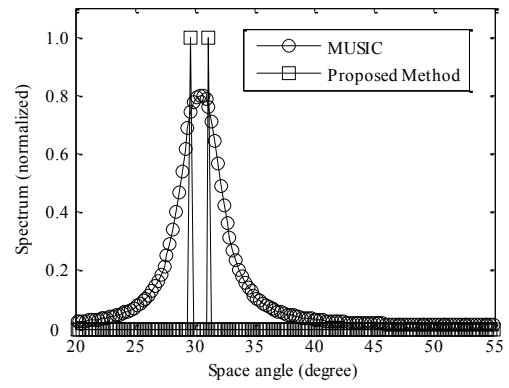

Fig. 4. Resolution performance comparison of space angles

As the azimuth and elevation angles is calculated by the estimated value of space angles, the estimation performance of 
azimuth and elevation is due to the estimation performance of space angle. Thus, high-resolution performance of space angles is necessary for the 2-D DOA estimation. From Fig. 4 we observe that the proposed method has narrower spectral peaks compared with the conventional MUSIC algorithm, which indicates that the proposed method has a better resolution performance than that of MUSIC algorithm.

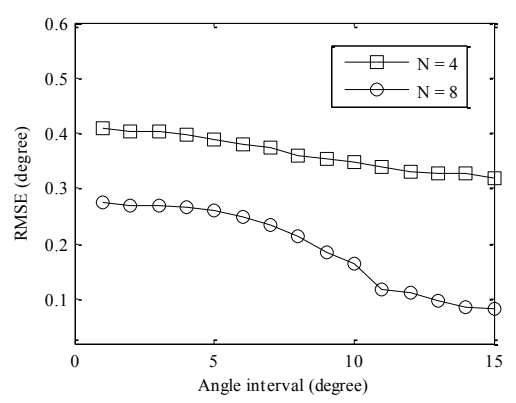

Fig. 5. RMSE of space angle under different angle interval

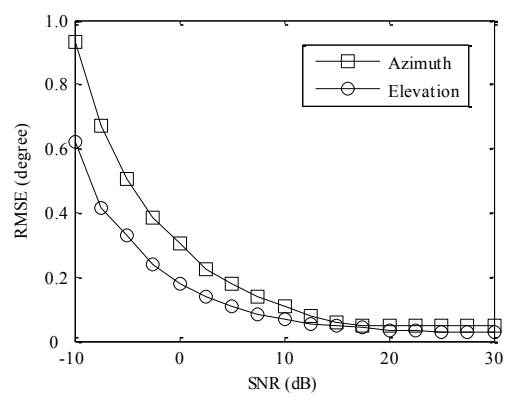

Fig. 6. RMSE of azimuth and elevation under different SNR

From the results of Fig. 5, we can see that the RMSE of space angle will decrease when increasing the angle interval and the number of sensors of sub-array. As the proposed method has a high-resolution performance of space angle, it can still achieve high estimation precision when the angle interval is small.

The results of Fig. 6 show that the RMSE of azimuth and elevation angle will decrease when the SNR varies from -10 $\mathrm{dB}$ to $30 \mathrm{~dB}$. Due to the excellent energy-concentrated performance of LFM signals in the fractional Fourier domain, we choose the peak values as the observed data for each sensor in sub-array $X$ and $Y$. Therefore, the proposed method can still achieve high estimation precision in low SNR.

\section{CONCLUSION}

The 2-D DOA estimation of LFM signals have been an active research in electronic reconnaissance. This paper has addressed the 2-D DOA estimation problem with sparse representation in fractional Fourier domain. Utilizing the energy-concentration properties of FRFT, the sparse recovery process is conducted in fractional Fourier domain. By defining the space angle, the proposed method reduces the dimension, which saves amount of computations. Moreover, the proposed method can work in broadband, multiple signals, and low SNR cases, which is suitable for electronic reconnaissance system.

\section{ACKNOWLEDGMENT}

This work was supported in part by the National Basic Research Program under Grants 61XX81, in part by the National Defense Pre-Research Program during the "12th FiveYear Plan" under Grants X110XX2030, and in part by the Fundamental Research Funds for the Central Universities of China under Grants K505120202.

\section{REFERENCES}

[1] J. E. Gonzalez, J.M. Pardo, A. Asensio and M. Burgos, "Digital signal generation for LFM-LPI radars,” Electron. Lett., vol. 39, no. 5, pp. 464 465, Mar. 2003

[2] R. O. Schmidt, "Multiple emitter location and signal parameter estimation," IEEE Trans. Antennas and Propagation, vol. 34, no. 3, pp. 276-280, Mar. 1986.

[3] R. Roy and T.Kailath, 'ESPRIT-Estimation of signal parameters via rotational invariance techniques," IEEE Trans. Acoust., Speech, Signal Process., vol. 37, no. 7, pp. 984-995, Jul. 1989.

[4] A. B. Gershman and M.G. Amin, "Wideband direction of multiple chirp signals using spatial time-frequency distributions," IEEE Signal Process. Lett., vol. 7, no. 6, pp. 152-155, Jun. 2000.

[5] H. M. Ozaktas, M. A. Kutay, and D. Mendlovic, "Introduction to the fractional Fourier transform and its applicactions," Advances in Imaging and Electron Physics, vol. 106, pp. 239-291, 1999.

[6] L. B. Almeida, "The fractional Fourier transform and time-frequency representations," IEEE Trans. Signal Process, vol. 42, no. 11, pp. 3084 3091, Nov. 1994.

[7] X.-M. Yang and R. Tao, "2-D DOA estimation of LFM signals based on fractional Fourier transform," Acta Electronica sinica, vol. 36, no. 9, pp. 1737-1740, Sep. 2008.

[8] J.-F. Gu, W.-P. Zhu, and M. N. S. Swamy, "Compressed sensing for DOA estimation with fewer receivers than sensors," Proc. IEEE Int. Conf. Circuits and Systems, 2011, pp. 1752-1755.

[9] Z. Liu, X. Wang, G. Zhao, G. Shi, and J. Lin, "Wideband DOA estimation based on sparse representation," Proc. IEEE Int. Conf. Signal process, Communication and Computing, 2013, pp. 1-4.

[10] D. M. J. Cowell and S. Freear, "Separation of overlapping linear frequency modulated (LFM) signals using the fractional fourier transform," IEEE Trans. Ultrason., Ferroelect., Freq. Control, vol. 57, no. 10, pp. 2324-2333, Oct. 2010.

[11] P. R. White and J. Locke, "Performance of methods based on the fractional Fourier transform for the detection of linear frequency modulated signals," IET Signal Process, vol. 6, no. 5, pp. 478-483, July, 2012.

[12] R. Chen and Y.-M. Wang, "Universal FRFT-based algorithm for parameter estimation of chirp signals," SIAM Journal on scientific computing, vol. 23, no. 4, pp. 495-501, Aug. 2012.

[13] E. J. Candes, J. Romberg, and T. Tao, "Robust uncertainty principles: exact signal reconstruction from high incomplete frequency information," IEEE Trans. Inf Theory, vol. 52, no. 2, pp. 489-509, Feb. 2006.

[14] E. J. Candes and T. Tao, "Near-optimal signal recovery from random projections: universal encoding strategies," IEEE Trans. Inf Theory, vol. 52, no. 12, pp. 5406-5425, Dec. 2006.

[15] S. S. Chen, D. L. Donoho, and M. A. Saunders, "Atomic decomposition by basis pursuit," SIAM Journal on scientific computing, vol. 20, no. 1, pp. 33-61, May. 1998.

[16] J. A. Tropp and A. C. Gilbert, "Signal recovery from random measurements via orthogonal matching pursuit," IEEE Trans. Inf Theory, vol. 53, no. 12, pp. 4655-4666, Dec. 2007. 